\title{
New bis-oxalamides from trans-1,2-diaminocyclohexane
}

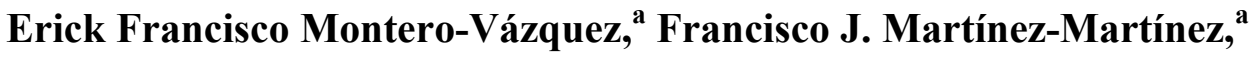 \\ Itzia I. Padilla-Martínez, ${ }^{\text {b }}$ M. Antonia Carvajal-García, a and Julio Hernández-Díaz * \\ ${ }^{a}$ Facultad de Ciencias Químicas, Universidad de Colima, Km 9 Carretera Colima-Coquimatlán, \\ Coquimatlán, Colima, México 28400 \\ ${ }^{b}$ Departamento de Química, Unidad Profesional Interdisciplinaria de Biotecnología del IPN, Av. \\ Acueducto s/n, Barrio La Laguna Ticomán, CP 07340, México D.F. \\ E-mail:julhed@ucol.mx
}

Dedicated to Professor Rosalinda Contreras on the occasion of her $60^{\text {th }}$ anniversary

\begin{abstract}
The synthesis of six new bis-oxalamides 2-7 derived from trans-1,2-diaminocyclohexane and aliphatic amines is reported. These compounds were characterized by IR, MS and ${ }^{1} \mathrm{H}$ and ${ }^{13} \mathrm{C}$ NMR spectroscopy.
\end{abstract}

Keywords: Bis-oxalamate, bis-oxalamide, macrocycle, ${ }^{1} \mathrm{H}$ and ${ }^{13} \mathrm{C}$ NMR spectroscopy

\section{Introduction}

Oxalamides are molecules that possess in their structure acidic protons and O-lone pairs which form inter- and intramolecular hydrogen bonds. Experimental and theoretical studies have demonstrated that intramolecular hydrogen bonds in oxalamides determine their geometry and conformation whereas intermolecular hydrogen bonds increase their stability. ${ }^{1-3}$ Due to these interactions, oxalamides are applied in diverse areas such as artificial receptors for biological recognition, ${ }^{4}$ in engineering and crystal design ${ }^{5}$ and in organogels formation. ${ }^{6}$ Recently, oxalamide derivatives were identified as HIV-1 inhibitors. ${ }^{7}$ Another important application of these compounds is in coordination chemistry as ligands. ${ }^{8}$

In this paper we report the synthesis and structural characterization by IR, MS and ${ }^{1} \mathrm{H}$ and ${ }^{13} \mathrm{C}$ NMR of six new oxalamides 2-7 derived from trans-1,2-diaminocyclohexane (Scheme 1). 


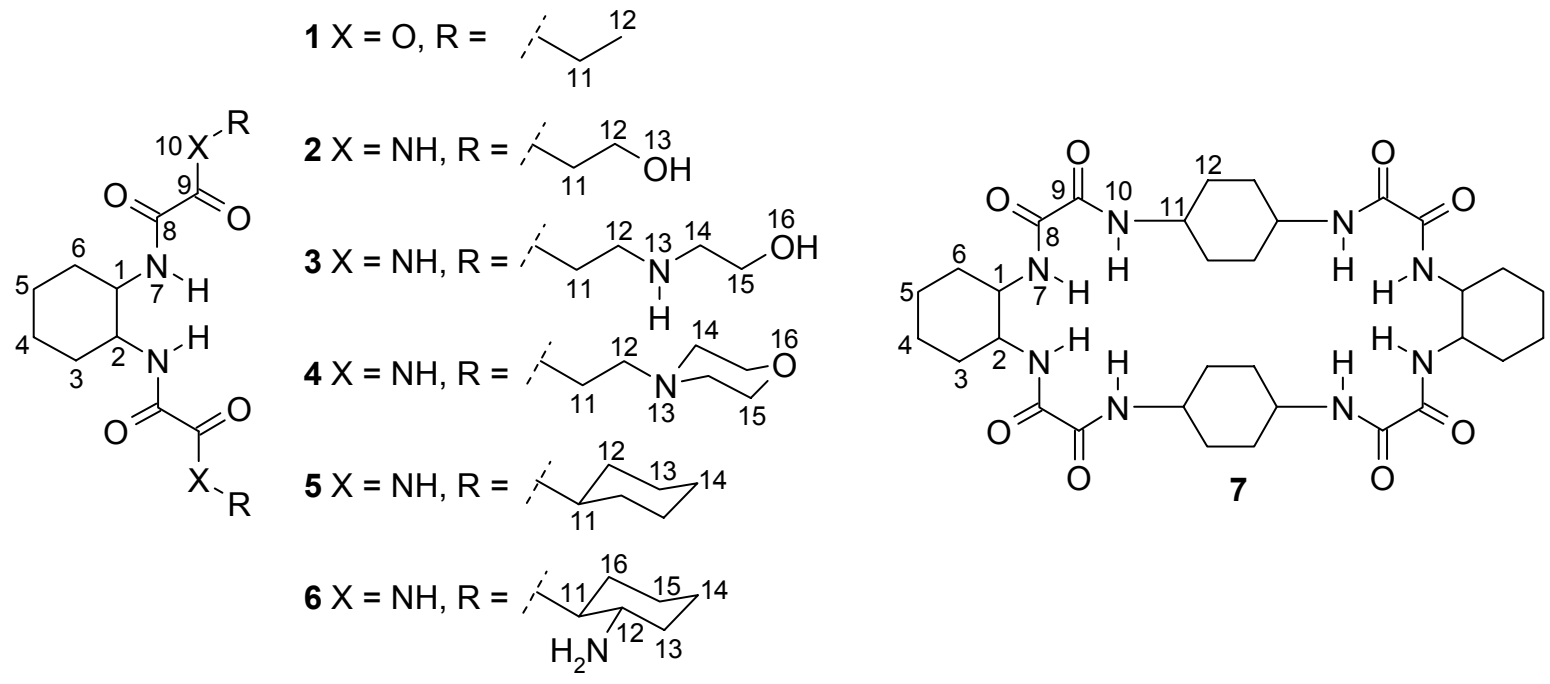

\section{Scheme 1}

\section{Results and Discussion}

\section{Synthesis}

Synthesis of bis-oxalamides 2-7 started with the preparation of the oxalamate 1 from condensation reaction of trans-1,2-diaminocyclohexane and ethyl chlorooxoacetate in the presence of $\mathrm{Et}_{3} \mathrm{~N}$ as catalyst, according to a procedure reported in the literature. ${ }^{9}$ Oxalamate 1 was first prepared by Albano and co-workers from enantiopure $(R, R)$ - and $(S, S)$-trans-1,2diaminocyclohexane. ${ }^{10}$ Spectroscopic data for oxalamate $\mathbf{1}$ determined in this study are similar to those reported by Albano, however we observed a melting point of $180-182^{\circ} \mathrm{C}$ which is $15^{\circ} \mathrm{C}$ higher than that reported. Condensation reaction of 1 and two equivalents of the corresponding alkylamines gave oxalamides 2-6. Under the same conditions $\mathbf{1}$ and trans-1,4diaminocyclohexane produced the macrocycle 7. Formation of 7 requires an excess of the diamine, no product formation was observed when the reaction was performed in an equimolar ratio. Macrocycles containing the oxalyl moiety are already known. ${ }^{11-13}$ Compounds 2-7 were analyzed in solution by ${ }^{1} \mathrm{H}$ and ${ }^{13} \mathrm{C}$ NMR spectroscopy using $\left[{ }^{2} \mathrm{H}\right] \mathrm{TFA}$ as solvent, because they were isolated as very insoluble solids.

\section{Infrared spectra}

The IR spectrum of 1 shows characteristic absorption bands at $3248(v \mathrm{~N}-\mathrm{H}), 1745(v \mathrm{O}=\mathrm{C}$ ester) and $1665 \mathrm{~cm}^{-1}$ ( $\mathrm{vO}=\mathrm{C}$ amide), in agreement with reported values ${ }^{10}$. For compounds 2-7 the IR spectra show one absorption band in the region of $3282-3276 \mathrm{~cm}^{-1}$ for the $v \mathrm{~N}-\mathrm{H}$ and only one strong band with an average value of $1644 \mathrm{~cm}^{-1}$ for $v \mathrm{O}=\mathrm{C}$ due to a similar connectivity in the oxalyl moiety. IR absorptions of 2-7 show a high frequency shift for $v \mathrm{~N}-\mathrm{H}$ and a low frequency 
shift for $v \mathrm{C}=\mathrm{O}$ with respect to $\mathbf{1}$, this behavior indicates that the electronic density of nitrogen is more engaged with carbonyl group in 2-7 than in 1. These values agree with reported data for similar compounds. ${ }^{9,10,14}$

\section{Mass spectra}

The analysis by mass spectrometry of compounds $\mathbf{4}$ and $\mathbf{5}$ showed the molecular ion, whereas $\mathbf{2}$, 3 and $\mathbf{6}$ showed the $[\mathrm{M}+1]^{+}$peak. Additionally $\mathbf{2}$ and $\mathbf{3}$ present the $[\mathrm{M}-\mathrm{OH}]^{+}$characteristic peak for a hydroxyl group. The molecular ion for compound 7 was not observed.

\section{NMR analysis}

The ${ }^{1} \mathrm{H}$ and ${ }^{13} \mathrm{C}$ NMR chemical shifts of compounds 1-7 are listed in Tables 1 and 2 respectively. The ${ }^{1} \mathrm{H}$ and ${ }^{13} \mathrm{C}$ NMR data determined for oxalamate $\mathbf{1}$ in this study are similar to those reported by Albano and co-workers. ${ }^{10}$ Intramolecular hydrogen bonding between N-H acidic protons and carbonyl oxygen atoms is known to favor the planar conformation and trans configuration of the oxalyl moiety. ${ }^{1}$ Because 2-7 were only soluble in $\left[{ }^{2} \mathrm{H}\right] \mathrm{TFA}$, which is a solvent that favors deuterium interchange, it was not possible to observe N-H chemical shifts and to conclude about hydrogen bonding in solution. The ${ }^{1} \mathrm{H}$ and ${ }^{13} \mathrm{C}$ NMR spectra of compounds 2-6 showed one half of the total expected signals because of the $C_{2}$ symmetry axis. H1 was observed as a broad signal between $3.91-4.00 \mathrm{ppm}$ and it is in the expected range. ${ }^{9} \mathrm{H} 3$ and $\mathrm{H} 4$ appeared as broad signals. The rigid conformation of 1,2-diaminocyclohexane ring for 1-6 in solution, allowed to distinguish equatorial $\mathrm{H} 3$ and $\mathrm{H} 4$ from axial $\mathrm{H} 3$ and $\mathrm{H} 4$ at room temperature. We were able to observe that the pendant arm of oxalamate 1 displayed a triplet for methyl protons and a highly symmetric 14 lines multiplet for the methylene protons, in contrast to the quartet triplet multiplicity reported by Albano and co-workers ${ }^{10}$ and for the analog oxalamate derived from trans-1,4-diaminocyclohexane. ${ }^{9}$ In our case, the multiplicity of methylene protons indicates that they have a different chemical environment, probably as a result of slow or no rotation of the pendant arm. The same behavior was observed for compound $\mathbf{2}$ and is equally expected for 3-6 because they gave broad signals. On the other hand, ${ }^{13} \mathrm{C}$ chemical shifts for 2-6 are in the characteristic range for this kind of compounds. ${ }^{9,10,15}$

A macrocyclic structure was proposed for compound 7 because its ${ }^{13} \mathrm{C}$ NMR spectrum showed only seven signals, instead of the nine expected if only one $\mathrm{NH}_{2}$ of trans-1,4diaminecyclohexane had reacted to give an open structure like that showed by 2-6. In the ${ }^{1} \mathrm{H}$ spectrum, compound 7 displayed only six broad signals, which fully correlated with ${ }^{13} \mathrm{C}$ NMR signals in the HETCOR spectrum. ${ }^{1} \mathrm{H}$ and ${ }^{13} \mathrm{C}$ NMR chemical shifts of 7 are similar to those determined for 2-6.

In future work, we will use the bis-oxalamides reported here as ligands in coordination chemistry. 
Table 1. ${ }^{1} \mathrm{H}$ NMR chemical shifts of compounds $1\left(\mathrm{CDCl}_{3}\right)$ and 2-7 in $\left[{ }^{2} \mathrm{H}\right] \mathrm{TFA}$

\begin{tabular}{ccccccl}
\hline Compd. & $\mathrm{H} 1, \mathrm{H} 2$ & $\begin{array}{c}\mathrm{H} 3, \mathrm{H} 6 \\
(\mathrm{eq})\end{array}$ & $\begin{array}{c}\mathrm{H} 3, \mathrm{H} 6 \\
(\mathrm{ax})\end{array}$ & $\begin{array}{c}\mathrm{H} 4, \mathrm{H} 5 \\
(\mathrm{eq})\end{array}$ & $\begin{array}{c}\mathrm{H} 4, \mathrm{H} 5 \\
(\mathrm{ax})\end{array}$ & \multicolumn{1}{c}{$\mathrm{R}$} \\
\hline $\mathbf{1}$ & $3.81, m$ & $2.08, m$ & $1.37, m$ & $1.82, m$ & $1.37, m$ & $\begin{array}{l}7.39, d,{ }^{3} J 6.14, \mathrm{NH} ; 4.33, m, \mathrm{H} 11 ; \\
\end{array}$ \\
& & & & & $1.37, d d,{ }^{3} J 7.09,{ }^{3} J 7.32, \mathrm{H} 12$ \\
$\mathbf{2}$ & $3.91, m$ & $2.07, m$ & $1.58, m$ & $1.90, m$ & $1.43, m$ & $3.61, m, \mathrm{H} 11 ; 3.97, t,{ }^{3} J 4.99, \mathrm{H} 12$ \\
$\mathbf{3}$ & $3.96, m$ & $2.09, m$ & $1.55, m$ & $1.89, m$ & $1.43, m$ & $3.73, m, \mathrm{H} 11 ; 3.58, m, \mathrm{H} 12 ; 3.50, m, \mathrm{H} 14 ;$ \\
& & & & & & $4.17, m, \mathrm{H} 15$
\end{tabular}

Table 2. ${ }^{13} \mathrm{C}$ NMR chemical shifts and peak multiplicities of compounds $\mathbf{1}\left(\mathrm{CDCl}_{3}\right)$ and $\mathbf{2 - 7}$ in $\left[{ }^{2} \mathrm{H}\right] \mathrm{TFA}$

\begin{tabular}{ccccccl}
\hline Compd. & $\mathrm{C} 1, \mathrm{C} 2$ & $\mathrm{C} 3, \mathrm{C} 6$ & $\mathrm{C} 4, \mathrm{C} 5$ & $\mathrm{C} 8$ & $\mathrm{C} 9$ & \multicolumn{1}{c}{$\mathrm{R}$} \\
\hline $\mathbf{1}$ & $53.9, d$ & $32.1, t$ & $24.6, t$ & $157.2, s$ & $160.4, s$ & $63.5, t, \mathrm{C} 11 ; 14.2, q, \mathrm{C} 12$ \\
$\mathbf{2}$ & $55.5, d$ & $31.9, t$ & $24.8, t$ & $160.8, s$ & $161.5, s$ & $43.0, t, \mathrm{C} 11 ; 61.7, t, \mathrm{C} 12$ \\
$\mathbf{3}$ & $55.8, d$ & $32.2, t$ & $24.9, t$ & $160.9, s$ & $162.7, s$ & $\begin{array}{l}38.2, t, \mathrm{C} 11 ; 49.3, t, \mathrm{C} 12 ; 51.5, t, \mathrm{C} 14 ; 58.8, \\
\end{array}$ \\
& & & & & $t, \mathrm{C} 15$ \\
$\mathbf{4}$ & $55.5, d$ & $32.1, t$ & $24.6, t$ & $160.6, s$ & n.o. $^{\mathrm{a}}$ & $\begin{array}{l}35.6, t, \mathrm{C} 11 ; 58.2, t, \mathrm{C} 12 ; 54.1, t, \mathrm{C} 14 ; 65.1, \\
\end{array}$ \\
& & & & & $t, \mathrm{C} 15$ \\
$\mathbf{5}$ & $55.7, d$ & $31.9, t$ & $24.8, t$ & $159.9, s$ & $161.5, s$ & $52.0, d, \mathrm{C} 11 ; 32.9, t, \mathrm{C} 12 ; 25.3, t, \mathrm{C} 13 ;$ \\
& & & & & & $25.7, t, \mathrm{C} 14$ \\
$\mathbf{6}$ & $54.7, d$ & $29.6, t$ & $23.0, t$ & $159.6, s$ & $160.8, s$ & $52.7, d, \mathrm{C} 11 ; 56.2, d, \mathrm{C} 12 ; 30.9, t, \mathrm{C} 13 ;$ \\
& & & & & & $23.5, t, \mathrm{C} 14 ; 23.5, t, \mathrm{C} 15 ; 30.9, t, \mathrm{C} 16$ \\
$\mathbf{7}$ & $55.2, d$ & $31.6, t$ & $24.3, t$ & $159.9, s$ & $160.9, s$ & $50.0, d, \mathrm{C} 11 ; 30.5, t, \mathrm{C} 12$ \\
\hline
\end{tabular}

${ }^{a}$ not observed. 


\section{Experimental Section}

General Procedures. Melting points were determined on a Melt Temp II apparatus in an open capillary tube and were not corrected. IR spectra were recorded in a Varian 3100 FT-IR Excalibur Series spectrometer equipped with an ATR device in the range of $400-4000 \mathrm{~cm}^{-1} .{ }^{1} \mathrm{H}$ and ${ }^{13} \mathrm{C}$ NMR spectra were recorded in a Varian Mercury $300\left({ }^{1} \mathrm{H}, 300.08 ;{ }^{13} \mathrm{C}, 75.46 \mathrm{MHz}\right)$ spectrometer in $\mathrm{CDCl}_{3}$ and $\left[^{2} \mathrm{H}\right] \mathrm{TFA}$ solution following standard techniques, chemical shifts are given in ppm and referred to $\mathrm{SiMe}_{4}$ as internal reference. Assignments of ${ }^{1} \mathrm{H}$ and ${ }^{13} \mathrm{C}$ signals were made on the basis of HETCOR experiments and by comparison to the reported values for similar compounds when possible. ${ }^{13} \mathrm{C}$ peak multiplicities were determined by APT experiments. The mass spectra were recorded on a Hewlett-Packard HP 5989A, EI MS, $70 \mathrm{eV}$. Elemental analyses were carried out in a Flash 1112 Thermo Finnigan analyzer.

Materials. Triethylamine (TEA), tetrahydrofuran (THF), trans-1,2-diaminocyclohexane, trans1,4-diaminocyclohexane, ethyl chlorooxoacetate, ethanolamine, cyclohexylamine, 2-(2aminoethylamino)ethanol and 4-(2-aminoethyl)morpholine, were purchased from commercial suppliers and used as received.

Diethyl $\boldsymbol{N}, \boldsymbol{N}^{\prime}$-cyclohexane-1,2-diyldioxalamate (1). trans-1,2-Diaminocyclohexane (1.05 $\mathrm{ml}, 1$ g, $8.75 \mathrm{mmol})$ and TEA $(2.44 \mathrm{ml}, 1.77 \mathrm{~g}, 17.51 \mathrm{mmol})$ in THF $(40 \mathrm{ml})$ were treated dropwise under vigorous stirring with ethyl chlorooxoacetate $(1.94 \mathrm{ml}, 2.39 \mathrm{~g}, 17.51 \mathrm{mmol})$ at $0{ }^{\circ} \mathrm{C}$. The reaction mixture was additionally stirred for $4 \mathrm{~h}$ at $25{ }^{\circ} \mathrm{C}$. The suspension was filtered and the solid was washed with water. THF solution was evaporated to dryness, washed with water, mixed with the previously obtained solid and dried to give $1(1.845 \mathrm{~g}, 67 \%)$ as a white solid. m.p. $180-182{ }^{\circ} \mathrm{C}$ (literature $157-165^{\circ} \mathrm{C}^{10}$ ). IR $v_{\max }\left(\mathrm{cm}^{-1}\right)$ (s, strong; m, medium; w, weak; br, broad): $3248(\mathrm{~N}-\mathrm{H}, \mathrm{m}) ; 2937,2867(\mathrm{C}-\mathrm{H}, \mathrm{w}) ; 1745,1665(\mathrm{C}=\mathrm{O}, \mathrm{s}) ; 1197(\mathrm{O}=\mathrm{C}-\mathrm{O}, \mathrm{s}) ; 1525(\delta$ $\mathrm{N}-\mathrm{H}, \mathrm{s}) . \mathrm{MS}, \mathrm{m} / \mathrm{e}(\%)$ : $[\mathrm{M}+1]^{+} 315.15$ (8), $\mathrm{M}^{+} 314.15$ (3), 241.20 (84), 197.20 (87), 167.05 (100), 124.05 (45), 81.15 (43).

\section{General synthesis of compounds 2-7}

Compounds 3-7 were synthesized according to the procedure described for 2 .

$N$-(2-Hydroxy-ethyl)- $N^{\prime}$-\{2-[(2-hydroxy-ethylaminooxalyl)-amino]-cyclohexyl\}-oxalamide

(2). $1(1 \mathrm{~g}, 3.18 \mathrm{mmol})$ and TEA $(0.88 \mathrm{ml}, 0.644 \mathrm{~g}, 6.36 \mathrm{mmol})$ in THF $(20 \mathrm{ml})$ were treated dropwise under vigorous stirring with ethanolamine $(0.38 \mathrm{ml}, 0.38 \mathrm{~g}, 6.36 \mathrm{mmol})$ at $25^{\circ} \mathrm{C}$. After refluxing for $5 \mathrm{~h}$, the solid was filtered and washed with hot THF $(5 \mathrm{ml})$ to give $2(0.8286 \mathrm{~g}, 75$ \%) as a white solid. m.p. $286-287{ }^{\circ} \mathrm{C}$. Anal. Calcd. for $\mathrm{C}_{14} \mathrm{H}_{24} \mathrm{~N}_{4} \mathrm{O}_{6}$ : C, 48.83; H, 7.02; N, 16.27 . Found: C, 48.59; H, 7.22; N, 15.99; IR $v_{\max }\left(\mathrm{cm}^{-1}\right): 3280(\mathrm{~N}-\mathrm{H}, \mathrm{m})$; 3200-3000 (O-H, br); 2931, $2859(\mathrm{C}-\mathrm{H}, \mathrm{w}) ; 1643(\mathrm{C}=\mathrm{O}, \mathrm{s}) ; 1513(\delta \mathrm{N}-\mathrm{H}, \mathrm{s}) . \mathrm{MS}, \mathrm{m} / \mathrm{e}(\%):[\mathrm{M}+1]^{+} 345.30(8),[\mathrm{M}-\mathrm{OH}]^{+}$ 327.30 (4), 314.20 (72), 256.20 (86), 238.20 (100), 212.15 (81), 184.20 (72), 167.15 (27), 141.20 (45), 97.20 (44), $81.15(38)$. 
$N$-[2-(2-Hydroxy-ethylamino)-ethyl]- $N^{\prime}$-(2-\{[2-(3-hydroxy-propylamino)-ethylaminooxalyl]amino\}-cyclohexyl)-oxalamide (3). 1 (1 g, $3.18 \mathrm{mmol})$, TEA (0.88 ml, $0.644 \mathrm{~g}, 6.36 \mathrm{mmol})$ and 2-(2-aminoethylamino)ethanol $(0.64 \mathrm{ml}, 0.66 \mathrm{~g}, 6.36 \mathrm{mmol})$ were refluxed for $3 \mathrm{~h}$. Product 3 (1.36 g, quantitative) was isolated as a white solid. m.p. 209-210 ${ }^{\circ} \mathrm{C}$. Anal. Calcd. for $\mathrm{C}_{18} \mathrm{H}_{34} \mathrm{~N}_{6} \mathrm{O}_{6} \cdot 0.5 \mathrm{H}_{2} \mathrm{O}$ : C, 49.19; H, 8.03; N, 19.12. Found: C, 49.38; H, 8.26; N, 19.13; IR $v_{\max }$ $\left(\mathrm{cm}^{-1}\right)$ : $3276(\mathrm{~N}-\mathrm{H}, \mathrm{m}) ; 3200-3000(\mathrm{O}-\mathrm{H}, \mathrm{br}) ; 2928,2832(\mathrm{C}-\mathrm{H}, \mathrm{w}) ; 1644(\mathrm{C}=\mathrm{O}, \mathrm{s}) ; 1512(\delta \mathrm{N}-\mathrm{H}$, s). MS, m/e (\%): $[\mathrm{M}+1]^{+} 431.35$ (3), $[\mathrm{M}-\mathrm{OH}]^{+} 413.30$ (2), 399.30 (10), 381.30 (22), 326.25 (54), 199.20 (39), 142.15 (26), 97.15 (29), 74.15 (100).

$\mathrm{N}$-(2-Morpholin-4-yl-ethyl)- $\mathrm{N}^{\prime}$-\{2-[(2-morpholin-4-yl-ethylaminooxalyl)-amino]-cyclohexyl\} -oxalamide (4). 1 (1 g, $3.18 \mathrm{mmol})$, TEA (0.88 ml, $0.644 \mathrm{~g}, 6.36 \mathrm{mmol})$ and 4-(2aminoethyl)morpholine $(0.82 \mathrm{ml}, 0.82 \mathrm{~g}, 6.36 \mathrm{mmol})$ were refluxed for $7 \mathrm{~h}$. Product $4(0.99 \mathrm{~g}$, $65 \%$ ) was isolated as a white solid. m.p. $260-262{ }^{\circ} \mathrm{C}$. Anal. Calcd. for $\mathrm{C}_{22} \mathrm{H}_{38} \mathrm{~N}_{6} \mathrm{O}_{6}$ : C, 54.76; $\mathrm{H}$, 7.94; N, 17.41. Found: C, 54.74; H, 8.15; N, 17.31; IR $v_{\max }\left(\mathrm{cm}^{-1}\right): 3282(\mathrm{~N}-\mathrm{H}, \mathrm{m}) ; 2939,2859$ $(\mathrm{C}-\mathrm{H}, \mathrm{w}) ; 1644(\mathrm{C}=\mathrm{O}, \mathrm{s}) ; 1117$ (C-O-C, m); 1514 ( $\delta \mathrm{N}-\mathrm{H}, \mathrm{s}) . \mathrm{MS}, \mathrm{m} / \mathrm{e}(\%): \mathrm{M}^{+} 482.45(6)$, 452.40 (6), 157.05 (3), 100.05 (100).

$\boldsymbol{N}$-Cyclohexyl- $\boldsymbol{N}^{\prime}$-[2-(cyclohexylaminooxalyl-amino)-cyclohexyl]-oxalamide (5). 1 (1 g, 3.18 mmol), TEA $(0.88 \mathrm{ml}, 0.644 \mathrm{~g}, 6.36 \mathrm{mmol})$ and cyclohexylamine $(0.72 \mathrm{ml}, 0.63 \mathrm{~g}, 6.36 \mathrm{mmol})$ were refluxed for $7 \mathrm{~h}$. Product 5 (1.24 g, $93 \%$ ) was isolated as a white solid. m.p. 297-301 ${ }^{\circ} \mathrm{C}$. Anal. Calcd. for $\mathrm{C}_{22} \mathrm{H}_{36} \mathrm{~N}_{4} \mathrm{O}_{4} \cdot 0.5 \mathrm{H}_{2} \mathrm{O}: \mathrm{C}, 61.51 ; \mathrm{H}, 8.68 ; \mathrm{N}, 13.04$. Found: $\mathrm{C}, 61.89 ; \mathrm{H}, 8.94 ; \mathrm{N}$, 12.97; IR $v_{\max }\left(\mathrm{cm}^{-1}\right): 3287(\mathrm{~N}-\mathrm{H}, \mathrm{m}) ; 2932,2856(\mathrm{C}-\mathrm{H}, \mathrm{m}) ; 1645(\mathrm{C}=\mathrm{O}, \mathrm{s}) ; 1513(\delta \mathrm{N}-\mathrm{H}, \mathrm{s})$. MS, m/e (\%): $\mathrm{M}^{+} 420.35$ (3), 339.25 (14), 294.15 (94), 250.20 (73), 222.20 (36), 167.15 (67), 141.15 (25), 97.15 (100), 81.15 (19).

$\mathrm{N}$-(2-Amino-cyclohexyl)- $N^{\prime}$-\{2-[(2-amino-cyclohexylaminooxalyl)-amino]-cyclohexyl $\}$ oxalamide (6). 1 (1 g, $3.18 \mathrm{mmol})$, TEA (0.88 ml, $0.644 \mathrm{~g}, 6.36 \mathrm{mmol})$ and trans-1,2diaminocyclohexane $(0.76 \mathrm{ml}, 0.72 \mathrm{~g}, 6.36 \mathrm{mmol})$ were refluxed for $7 \mathrm{~h}$. Product $6(1.30 \mathrm{~g}$, quantitative) was isolated as a white solid. m.p. above $340{ }^{\circ} \mathrm{C}$. Anal. Calcd. for $\mathrm{C}_{22} \mathrm{H}_{38} \mathrm{~N}_{6} \mathrm{O}_{4} \cdot 1.7 \mathrm{H}_{2} \mathrm{O}$ : C, 54.91; H, 8.60; N, 17.46. Found: C, 55.19; H, 8.53; N, 17.05; IR $v_{\max }$ $\left(\mathrm{cm}^{-1}\right): 3275(\mathrm{~N}-\mathrm{H}, \mathrm{m}) ; 2925,2855(\mathrm{C}-\mathrm{H}, \mathrm{w}) ; 1643(\mathrm{C}=\mathrm{O}, \mathrm{s}) ; 1506(\delta \mathrm{N}-\mathrm{H}, \mathrm{s}) . \mathrm{MS}, \mathrm{m} / \mathrm{e}(\%)$ : $[\mathrm{M}+1]^{+} 451.45$ (2), 354.30 (89), 186.25 (10), 97.15 (100), 71.15 (25), 42.15 (54).

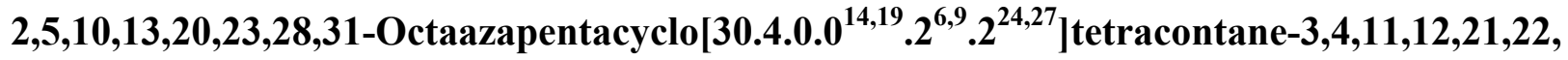
29,30-octaone (7). 1 ( $1 \mathrm{~g}, 3.18 \mathrm{mmol})$, TEA $(0.88 \mathrm{ml}, 0.644 \mathrm{~g}, 6.36 \mathrm{mmol})$ and trans-1,4diaminocyclohexane $(0.72 \mathrm{~g}, 6.36 \mathrm{mmol})$ were refluxed for $2 \mathrm{~h}$. Product 7 (1.05 g, quantitative) was isolated as a white solid. m.p. above $340{ }^{\circ} \mathrm{C}$. Anal. Calcd. for $\mathrm{C}_{32} \mathrm{H}_{48} \mathrm{~N}_{8} \mathrm{O}_{8} \cdot 3.5 \mathrm{H}_{2} \mathrm{O}: \mathrm{C}, 52.23$; H, 7.53; N, 15.23. Found: C, 52.40; H, 7.90; N, 15.15; IR $v_{\max }\left(\mathrm{cm}^{-1}\right): 3278(\mathrm{~N}-\mathrm{H}, \mathrm{m}) ; 2931$, $2859(\mathrm{C}-\mathrm{H}, \mathrm{w}) ; 1644(\mathrm{C}=\mathrm{O}, \mathrm{s}) ; 1502(\delta \mathrm{N}-\mathrm{H}, \mathrm{s}) . \mathrm{MS}, \mathrm{m} / \mathrm{e}(\%): 168.15(10), 141.15(18), 113.05$ (14), 97.05 (55), 82.15 (13), 71.15 (20), 58.15 (100), 43.15 (53). 


\section{Acknowledgements}

This work was supported by PROMEP-México and Fondo Ramón Álvarez-Buylla de Aldana 368/05, Universidad de Colima. E. F. M.-V. thanks Conacyt-México for a scholarship.

\section{References}

1. Martínez-Martínez, F. J.; Padilla-Martínez, I. I.; Brito, M. A.; Geniz, E. D.; Rojas, R. C.; Saavedra, J. B. R.; Höpfl, H.; Tlahuextl, M.; Contreras, R. J. Chem. Soc., Perkin Trans 2 1998, 401.

2. Aleman, C.; Casanovas, J. J. Mol. Struct. 2004, 675, 9.

3. Desseyn, H. O.; Perlepes, S. P.; Clou, K.; Blaton, N.; Van der Veken, B. J.; Dommisse, R.; Hansen, P. E. J. Phys. Chem. 2004, 108, 5175.

4. Nowick, J. S.; Tsai, J. H.; Bui, Q.-C.; Maitra, S. J. Am. Chem. Soc. 1999, 121, 8409.

5. (a) Liu, Y.; Lam, A. H. W.; Fowler, F. W.; Lauher, J. W. Mol. Cryst. Liq. Cryst. 2002, 389, 39. (b) Curtis, S. M.; Le, N.; Fowler, F. W.; Lauher, J. W. Cryst. Growth Des. 2005, 5, 2313. (c) Nguyen T. L.; Scott A.; Dinkelmeyer, B.; Fowler, F.; Lauher J. W. New J. Chem. 1998, 129.

6. (a) Makarevic, J.; Jokic, M.; Raza, Z.; Caplar, V.; Katalenic, Z. S.; Kojic-Prodic, B.; Zinic, M. Croat. Chem. Acta. 2004, 77, 403. (b) Frkanec, L.; Jokic, M.; Makarevic, J.; Wolsperger, K.; Zinic, M. J. Am. Chem. Soc. 2002, 124, 9716.

7. McFarland, C.; Vivic, D. A.; Debnath, A. V. Synthesis 2006, 5, 807.

8. (a) Costa, L. C. M.; Maia, J. R. S.; De Lima, G. M.; Ardisson, J. D. Main Group Met. Chem. 2004, 27, 247. (b) Liu, Z.-L.; Li, L.-C.; Liao, D.-Z.; Jiang, Z.-H.; Yan, S.-P. Cryst. Growth Des. 2005, 5, 783.

9. Martínez-Martínez, F. J.; Maya-Lugardo, P.; García-Báez, E. V.; Höpfl, H.; Hernández-Díaz, J.; Padilla-Martínez, I. I. Acta Cryst. 2005, E 61, o2994.

10. Albano, V. G.; Bandini, M.; Monari, M.; Marcucci, E.; Piccinelli, F.; Umani-Ronchi, A. J. Org. Chem. 2006, 71, 6451.

11. Gao, E.-Q.; Liao, D.-Z.; Jiang, Z.-H.; Yan, S.-P. Polyhedron 2001, 20, 923.

12. Nishat, N.; Haq, M. M.; Siddiqi, K. S. Synth. React. Inorg. Met.-Org. Chem. 2001, 31, 1599.

13. Hechavarria-Fonseca, M.; Hjelmgaard, T; König, B. Molecules 2003, 8, 453.

14. Padilla-Martínez, I. I.; Martínez-Martínez, F. J.; Guillén-Hernández, C. I.; Chaparro-Huerta, M; Cabrera-Pérez, L. C.; Gómez-Castro, C. Z.; López-Romero, B. A.; García-Báez, E. V. ARKIVOC 2005, (vi), 401.

15. Low, J. N.; Milne, B. F.; Ross, J. N.; Wardell, J. L. J. Braz. Chem. Soc. 2002, 13, 207. 